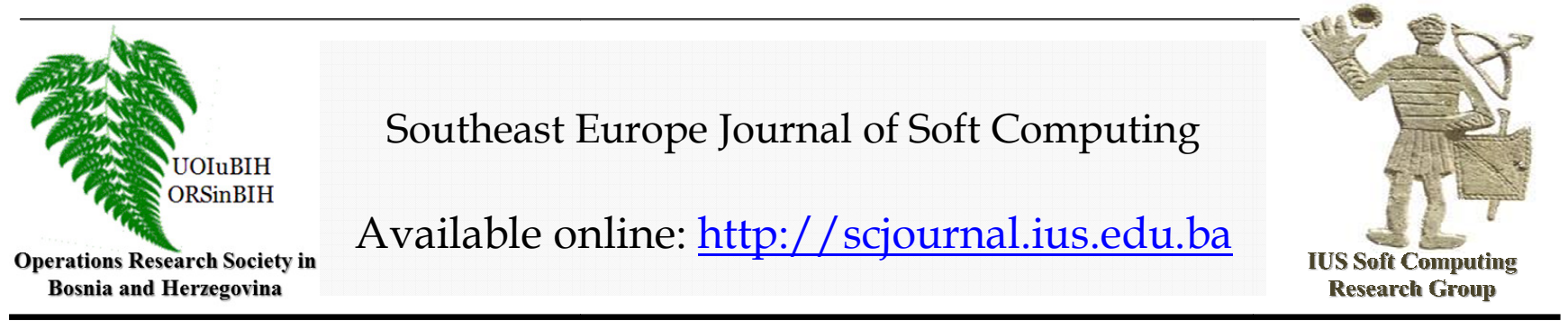

\title{
Structural Bioinformatics Analysis On Diabetes Associated Proteins
}

\author{
Elif Colak \\ International University of Sarajevo, \\ Faculty of Engineering and Natural Sciences, \\ HrasnickaCesta 15, Ilidža 71210 Sarajevo, \\ Bosnia and Herzegovina \\ elif-colak@hotmail.com
}

\section{Article Info}

Article history:

Article received on 10 Jan. 2016

Received in revised form 12 Feb.2016

\begin{abstract}
The Human Diabetes Proteome Project (HDPP) mentioned more than 1000 diabetes associated proteins. 400 diabetes associated proteins whose structure is not analyzed yet are selected from the database. Each proteins structure is analyzed via prediction tools in order to reveal structural similarity with IAPP (Islet Amyloid Polypeptide) whose role is well characterized in diabetes. Through similarity analysis between proteins and in region of disulfide bridge formation, we aimed to find similar possible misfolding patterns in other diabetes associated proteins. Result indicates that Calcitonin Gene Related Peptide I is the only proteins who shows high structural and conformational similarity with IAPP. We believe that one of the possible enrolments of the protein Calcitonin Gene Related Peptide 1 to the diabetes can be similar conformational changes due to disulfide bridge formation break. Finally we propose that the accumulation of misfolded Calcitonin Gene Related Peptide 1 is similar to other protein based conformational diseases. Further structural analyses needs to be performed to confirm these results.
\end{abstract}

Keywords:

Islet amyloid polypeptide, conformational disease, similarity analysis

\section{INTRODUCTION}

Insulin is an important anabolic hormone for carbohydrates, lipids, and proteins. So defects in insulin secretion or action can cause to metabolic abnormalities. Insufficient levels of insulin to perform its action and/or insulin resistance in the target tissues such as mainly adipose tissue, skeletal muscles, and partially liver, at the level of insulin receptors, signal transduction system, and/or effector enzymes or genes all are associated with these metabolic abnormalities [1-3].

Diabetes mellitus or diabetes is a set of diseases described as high level of blood glucose resulting from the bodies' inability of insulin secretion and/or insulin action.

The symptoms can vary due to the type and period of diabetes. Some of the diabetes patients are symptomless particularly patients with type 2 diabetes at the early stage of the disease, others were diagnosed with hyperglycemia. The disease absolute insulin deficiency in children may present with characteristic symptoms such as polyuria, polydipsia, polyphagia, weight loss, and blurred vision. When diabetes is uncontrolled because of appearance ketoacidosis or rare from nonketotic hyperosmolar syndrome, it can cause anesthesia, coma and even death if it is not cured[1-3].

\section{Pathophysiology of Diabetes mellitus}

To understand the pathophysiology of diabetes, the basics of insulin function on carbohydrate metabolism needs to be considered. Insulin is a polypeptide hormone, synthesized by the beta cells of the islets of Langerhans in the pancreas of humans and other mammals. $2 \%$ of the total mass of the pancreas consists of the islets of Langerhans, whereas $60-80 \%$ of all the cells of islets of Langerhans consist of beta cells [4]. 
The synthesis of carbohydrates, proteins, lipids, and nucleic acids are promoted by insulin hormone and those processes are known as the basic physiological function of insulin. Specific pathways such as stimulation of glucose transport across muscle and adipocyte cell membranes, regulation of hepatic glycogen synthesis, and inhibition of glycogenolysis and gluconeogenesis all are involved in carbohydrate metabolism which is affected by insulin [5]. Eventually by these actions, a blood glucose concentration is reduced.

The glucose homeostasis and the postprandial glucose load are regulated by insulin which mediates glucose uptake of cells [6]. The amount of insulin production is directly correlated with the amount of sugar intake. When more sugar is consumed, the tiny pancreatic beta cells are not able to produce enough level of insulin. The cells with a limited capacity which are forced to produce insulin fail to live whereas the other cells with a sufficient capacity can continue their lifetime under normal dietary conditions [7].

When insulin production and secretion are affected by the disease, blood glucose level will be also affected. When insulin production is reduced, glucose uptake of cells will be inhibited, resulting disease is called hyperglycaemia. In the other way, if insulin is secreted but not used properly by target cells, it will give same results. When insulin production is high, blood glucose levels will be very low (hypoglycemia) as thus, higher amounts of glucose enter the cells and lower remains in the bloodstream. Multiple hormones play a role in glycemia disease. While insulin is the only hormones that decrease glucose levels in blood, the counter-regulatory hormones such as glucagon, catecholamine, growth hormone, thyroid hormone, and glucocorticoids increase blood glucose levels [8].

\section{Diagnosis of Diabetes mellitus}

Several type of tests are available to diagnose diabetes mellitus such as the estimation of fasting plasma glucose test (FPG),oral glucose tolerance test (OGTT) or hemoglobin A1C (HbA1c). Evaluation of these tests is based on the correlation of FPG or HbAlc with retinopathy. HbA1c has been recommended to diagnose diabetes by the International Expert Committee in 2009 and approved by ADA, the Endocrine Society, the WHO and many scientists and related organizations all over the world[9,10,11].

\section{Types of Diabetes mellitus}

In 1997, the American Diabetes Association (ADA) categorized diabetes mellitus as type 1, type 2, other types and GDM (gestational diabetes mellitus). This classification is still the most common classification applied by ADA[1].Type 1 diabetes, also known as insulin dependent diabetes mellitus (IDDM), is describes as pancreatic beta cells inability to secrete insulin. Type 2 diabetes, also called non-insulin dependent diabetes mellitus (NIDDM), occurs when target tissues decrease the sensitivity to insulin. The reduction event of sensitivity for insulin is often considered insulin resistance. The efficient uptake and utilization of glucose by the body's cells, except those of the brain, is prevented by insulin deficiency or insulin resistance on glucose metabolism [12]. Consequently, blood glucose concentration increases and cell utilization of glucose falls as well utilization of fats and proteins increases [13].

\section{Type I Diabetes mellitus}

The destruction of $\beta$ cells of the pancreas generates type 1 diabetes mellitus by composing $5 \%-10 \%$ of patients diagnosed with diabetes $[14,15]$. Development of Type 1 diabetes mainly depends on destructed autoimmune system of the pancreatic $\beta$ cells during T-cell mediated inflammatory response (insulitis) and a humoral (B cell) response [15].A distinctive feature of type 1 diabetes is that auto antibodies against the pancreatic islet cells, although the role of pancreatic antibodies on the disease is not clear. The pancreatic auto antibodies can be detected as a characteristic of type I diabetes in the serum of diabetes patients months or years before the birth of the disease [16].

Type 1diabetes often emerges suddenly and shows symptoms such as polydipsia, polyuria, enuresis, lack of energy, extreme tiredness, polyphagia, sudden weight loss, slow-healing wounds, recurrent infections and blurred vision with severe dehydration and diabetic ketoacidosis in children and adolescents [17].

\section{Type II Diabetes mellitus}

A report published in 2013 by the IDF indicates that the global prevalence of diabetes in adults which are between 20-79 years old was $8.3 \%$ (382 million people), with 14 million more men than women (198 million men vs. 184 million women). Most of the patient between the ages 40 and 59 years and the number is expected to rise beyond 592 million by 2035 with a $10.1 \%$ global prevalence. Patient who are diagnosed currently with diabetes exceed half a billion while 175 million cases are still undiagnosed. More than $90 \%-95 \%$ of diabetes patients belong to type 2 and they are mostly adults [17].

\section{TYPE II DIABETES MELLITUS AS A CONFORMATIONAL DISEASE: CONFORMATIONAL APPROACH TO TYPE II DIABETES MELLITUS}

When an endogenous protein is changed by its shape it leads to development of conformational diseases in which self-association of proteins and tissue deposition occur [18]. In the normal process of protein biosynthesis, when misfolding occurs, the intracellular mechanisms have evolved to shuttle and degrade abnormal proteins[19]. Despite the fact that conformational changes occur in 
normal protein processing, a specific protein's tendency to aggregation, and a genetic or environmental predisposition to disease can suppress the quality control mechanisms of the cell. When proteins are unfolded, a cell responds to this change by dealing with reactive oxygen species and oxidative stress requires functional chaperones, antioxidant production, protein degradation and a cascade of intracellular events collectively. High concentrations level of mutated protein cause to aggregation and deposition into tissues over time slowly. These steps of events need a certain time to occur and can partially reveal the relatively late clinical presentation of many conformational diseases.

Frequently, in conformational diseases, a protein is characterized by aggregations in beta-sheet linkages. Alternated peptide strands that are linked by hydrogen bonds between their aligned pleated structures form betapleated sheets which are known a feature of the systemic amyloidoses, neurodegenerative diseases and type 2 diabetes mellitus (T2DM). The distinct clinical presentations of these diseases, as well as the fact that some of them are almost whollyarisen from genetic deficiencies for example Huntington's disease whereas others such as T2DM can be related tostrong environmental component like obesity, may seem to disprove the single grouping of them on the basis of conformational abnormalities. However, one benefit of the designation 'conformational disease' is that it implies the mechanisms underlying the sometimes bizarre and delayed presentation of these diseases. Although fibril formation is a characteristic of these diseases, they consist of aggregation of different proteins by sharing structural properties[20].

To treat T2DM as a conformational disease does not denotethat the disease can be completely defined as inappropriate protein aggregation. For explanation of disease pathogenesis entirely other associated processes, like insulin resistance, require other models. Like Alzheimer's disease in which brain amyloid shows the climax of several previous events and cannot perfectlydemonstrate cognitive defaults, therefore in T2DM, islet amyloid is both cause and consequence of several disease processes.

\section{Islet Amyloid Polypeptide (IAPP)}

Islet amyloid which was initially called "islet hyalinization" defined in 1901independently by two researchers and for a long time it was stayed as amystery [21]. It was found that it was associated with diabetes mellitus, particularly in elderly people, but importance of its possible pathogenicity was often denied, since it was not found in all individuals with diabetes [22]. It had been shown in 1959 that several amyloid types have an ultrastructural characteristic, and accumulated islets share this appearance [23]. Biochemical analyses of amyloid fibrils from systemic primary and secondary amyloidosesrevealed that they are consisted of distinctive proteins, therefore there was a doubt about that the islet deposits might also be a polymerized protein[22].

Even though the characteristics of other amyloid fibrils had been clarified, the chemical configuration of islet amyloid did not get considerable attention. The hormonal origin of amyloid polypeptidein C cell-derived medullary thyroid carcinoma indicates important point that amyloid in other endocrine tissues also comes from the local secretory products, and hence, it was considered that insulin, or proinsulin, or split products can be composed of the islet amyloid fibrils [24]. Immunological trials to analyze the amyloid yielded ambiguous results, and to purify a major fibril protein was prevented by greater insolubility of islet amyloid deposits when compared to other amyloid forms, by excluding the amyloid core in Alzheimer's plaques [25]. But when using concentrated formic acid on extracted amyloid from an amyloid-rich insulinoma, there is a possibility to purify the major fibril protein and characterize it by NH2-terminal amino acid sequence analysis in whicha novel peptide is revealedunexpectedly, not similar any part of proinsulin while with partial identity to the neuropeptide calcitonin gene-related peptide (CGRP). The length of the islet amyloid peptide as a37aminoacid(aa) residue was proved by further characterization of the peptide purified from an insulinoma and from islet amyloid of human andfelineorigin. The peptide was firstly called "insulinoma amyloid peptide", then diabetes-associated peptide (DAP), and finally islet amyloid polypeptide (IAPP), or "amylin" [26].

\section{Structure of Islet Amyloid Polypeptide}

IAPP is 37-amino acid in length and member of the calcitonin family. And also contains calcitonin, CGRP, adrenomedullin, and intermedin[27]. It is $43-46 \%$ identity between human IAPP and the two CGRPs [28]. As the other peptides in the family, IAPP occurs a disulfide bridge between amino acid residues 2 and 7 and an amidated $\mathrm{COOH}$ terminus (Figure 1). The disulfide bridges and amidated $\mathrm{COOH}$ terminus are involved in posttranslational modifications. They are essential for biological function. It was mentioned IAPP has a random coil structure [29]. On the other hand, both CD and NMR studies revealed that IAPP forms transient amphipathic helix in the NH2-terminal region [30,31](Figure 2). Exceptionally for the very NH2- terminal part which creates a rigid ring structure, due to the presence of disulfide bridge between residues 2 and 7[32].It was found unstructured part of the molecule is the $\mathrm{COOH}-$ terminal[30]. It was supposed that the helical part is crucial in receptor binding and can be also associated with the pathological transformation to amyloid fibrils [30]. 


\section{KCNTATCATQRLANFLVHSSNNFGAILSSTNVGSNTY-NH2}

Figure 1:Amino acid sequence of human-IAPP contains the disulfide bridge between Cys 2 - Cys 7 and amidated Cterminus.

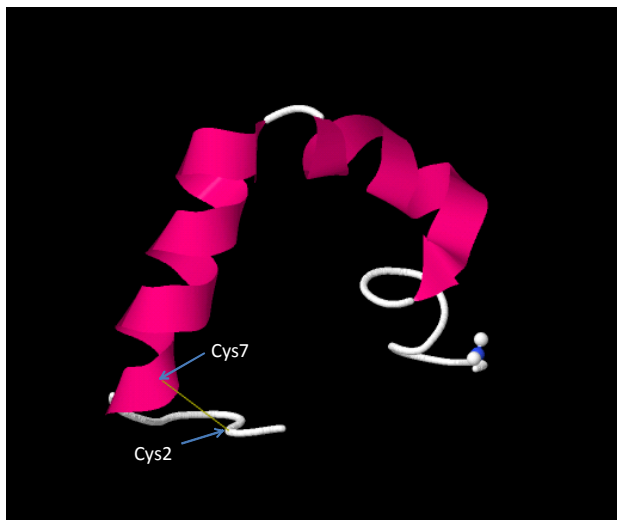

Figure 2: Representation of NMR structure of humanamyloid polypeptide by PDB.Blue arrows indicate cysteine residue 2 and 7 which form disulfide bridge.

\section{Expression of Islet Amyloid Polypeptide}

IAPP is a 37 -aa of peptide in length, but the application of molecular biological methods revealed that IAPP is expressed originally as part of an 89 -aa residue preprotein which consists of a 22-aa signal peptide and two short flanking peptides, the latter cleaved off at double basic aa residues similar to proinsulin [31]. One single-copy gene located on the short arm of chromosome 12 expresses IAPP, compared to insulin and the other members of the calcitonin family which involves CGRP, adrenomedullin, and calcitonin, they are all encoded by genes on the evolutionary related chromosome. The preproIAPP gene consists of three exons; the last two of them encode the complete prepromolecule[32]. The progression of proIAPP (67-aa residue) to IAPP occurs in the secretory vesicles with the cleavage of the signal peptide in the endoplasmic reticulum (ER). ProIAPP (Figure 3) and proinsulin are both converted by the two endoproteases prohormone convertase 2 (PC2) and prohormone convertase 1/3 $(\mathrm{PC} 1 / 3)$ and by carboxypeptidase E (CPE). This conversion depends on $\mathrm{pH}$ and happens in the late Golgi and secretory granules. PC2 cleavesproIAPP at position Lys10-Arg11, and PC1/3 at position Lys50-Arg51. It was reported that instead of $\mathrm{PC} 1 / 3, \mathrm{PC} 2$ is able to process proIAPP at the $\mathrm{COOH}$-terminal processing site [33]. The $\mathrm{COOH}$-terminal glycine residueused for amidation with an occurrence of disulfide bridges between residues 2 and 7 , is a prerequisite for whole biological activity of several other hormonal peptides. The $\mathrm{C}$ peptide from proinsulin and the two flanking peptides from proIAPP after removing process stay in the secretory granule, result of exocytosis by releasing of equimolar concentrations of the removed peptides and their respective final hormonal product.

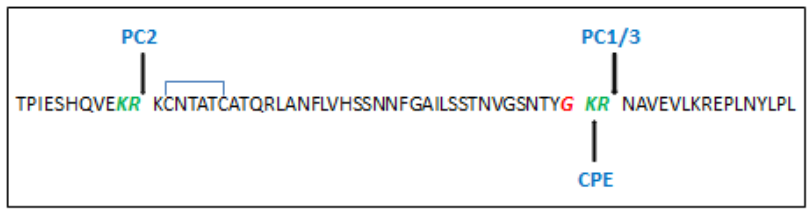

B

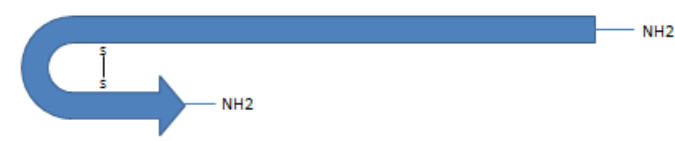

Figure 3: Cleavage process of proIAPP into IAPP. A indicates proIAPP,B indicates human-IAPP after cleavage.

IAPP and insulin genes have similar promoter, and the transcription factor PDX1 which controls the effects of glucose on both genes [34]. $\beta$-cells stimulated by glucose respond as a parallel secretion of IAPP and insulin in the rat but, this parallel secretion of IAPP and insulin is modified in experimental diabetes models in rodents [35]. In human recipients with transplanted islets in the liver to become insulin-independent, show disproportionately more IAPP than normal secreted during hyperglycemia [36]. These examples reveal that the possible disturbance of strictly parallel expression of IAPP and insulin under certain conditions. IAPP is expressed not only in the islet $\beta$-cells but also in the $\delta$-cells in rat and mouse; in the gastrointestinal tract of the rat, mouse, cat, and human and in sensory neurons in rats [37].

\section{Physiology and Pathophysiology Effects of Islet Amyloid Polypeptide}

The function of IAPP is not perfectly clear due to the difficulty in discriminating between its physiological or pathophysiological role and its pharmacological effects experimented successfully. This cannot be happen always. IAPP is secreted with insulin in the secretory vesicles, and it supported that it plays a role in the regulation of glucose metabolism. At the early stage of the discovery of IAPP, it was revealed that insulin-stimulated glucose uptake and glycogen synthesis can be inhibited by the peptide in extracted incubated rat skeletal muscle [38]. This inhibition may be mediated by effects on several enzymes [39]. It was also found that a post-insulin-receptor effect of IAPP prevents insulin-stimulated glucose transport in vitro [40]. It was primarily assumed that the insulin resistance 
mechanism in type 2 diabetes had been shown with the discovery of IAPP, and it was found that insulin resistance could be activated by infusion of IAPP in vivo [41]. However, these effects were carried out at concentrations much higher than those seen physiologically and therefore they were considered as pharmacological rather than physiological. Higher plasma concentration IAPP associated with renal insufficiency does not effect to insulin secretion [42]. After all, the possible role of IAPP in the alteration of insulin effects on peripheral tissues is still debatable, and it cannot be excluded that IAPP has an important function in the fine controlling, that is difficult to prove experimentally. Nowadays, there are two logical physiological roles of IAPP that can be interested in particularly. One of them is its function as an auto- or paracrine molecule in the islets of Langerhans, while the other is its role as a hormone with effects on the central nervous system.

\section{Islet Amyloid Formation and Conformational Change in T2DM}

Amyloid is a general term used for a particular protein aggregation condition in which molecules in $\beta$-sheet conformation are bound together predominantly by hydrogen bonds and also by other bonds [43]. This aggregation event creates $10 \mathrm{~nm}$ thin, stable fibrils which oriented the $\beta$-strands perpendicular to the fibril axis. In humans, there are more than 25 proteins which are shown to form amyloid fibrils, and more will be analyzed [44]. Between most of them there is no relation, and at this moment it is not known why only particular proteins aggregation occurs. Possibly a small size, low degree of native secondary structure and local occurrence at high concentration make polypeptide hormones overrepresented[45]. Many numbers of small peptides and proteins can form fibrils in vitro with amyloid-like properties [43]. However, the designated amyloid should be conserved for the material deposition in vivo, with its fibril core protein, has components including proteoglycans and usually the glycoprotein serum amyloid $\mathrm{P}$ component [44]. So, fibrils in vitro are not exactly same with amyloid but "amyloid-like" [46].

Addition to IAPP and also many other polypeptide hormones can be deposited as amyloid, and they are commonly found in endocrine tumors [47]. Insulin is also shown as an islet amyloid fibril protein; it does not occur spontaneously in humans, interestingly, it has been observed in the hystricomorphic rodent degu [48]. The association of insulin-derived islet amyloid with diabetes in the degu extremely indicates the importance of islet amyloid in human type 2 diabetes. In humans, insulin aggregation into an iatrogenic form of amyloidosis can be occurred at the sites of injections[49].

As an expectation from a polypeptide hormone, IAPP is preserved along evolution, and the molecule has been determined in mammals, birds, and teleostanfishes [50]. Particularly, the NH2- and $\mathrm{COOH}$-terminal parts of IAPP are conserved (Figure 4). The pathological state of IAPPderived amyloid in the islets of Langerhans occurs in some other mammalian species as well as occurs in humans. One plausible reason for the late occurred interest in islet amyloid may be due to the fact that it does not present in the animals commonly used in diabetes research, such as the rat and mouse [51]. Regarding to this notion rat and mouse IAPP which are identical, lack fibrillogenicity in vivo and in vitro. The reason for this is to be found in differences in the primary structure. In addition, IAPP contains a conserved region; there is an interspecies difference at some critical amino acid residues. Most clear ones are the variations detected in the IAPP20-29 region, where five out of six differences between rat and human IAPP are observed (Figure 4). Remarkably, in this region rat/mouse IAPP has three proline residues which are shown as $\beta$-sheet breakers. A synthetic human IAPP20-29 is highly fibrillogenic, whereas the corresponding rat peptide is not [52]. Addition to the rat, several rodents also shares this sequence characteristic, while species with amyloidogenic IAPP involve humans, nonhuman primates, and cats [53]. These variations just clarify either why amyloid can develop or why it does not.

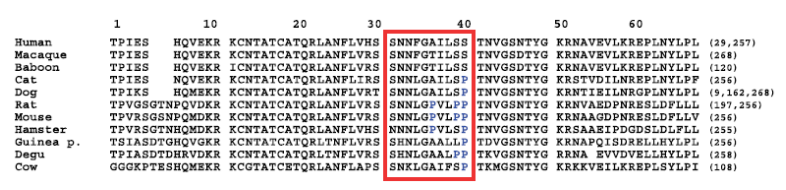

Figure 4:The proIAPP amino acid sequences of the human with some mammalian species. The phylogenetically conserved region of IAPP is drawn with a red box[54].

Later researches have demonstrated that along with the IAPP20-29 region is crucial to amyloid formation, other parts of the molecule are also important in fibrillogenesis[55]. The amphipathic helical region of the molecule involves IAPP14-20 domain which can be of particular interest [30,56].

\section{Conformational Change of IAPP in T2DM}

The presence of islet amyloidosis to disease pathogenesis has been forcefullyseen as an argument [57]. Islet amyloid polypeptide (IAPP) oligomers that lead islet amyloid deposition are likely more poisonous to beta cells than islet amyloid itself [58]. Autopsy results of $96 \%$ of patients with T2DM show the presence of islet amyloid[59]. The accumulations of harmful, mis-folded IAPP oligomers and deposition of crossed beta-pleated sheets make T2DM is similar to other protein based conformational diseases. The human islet amyloid polypeptide (hIAPP) is exhibited in Figure 5. 


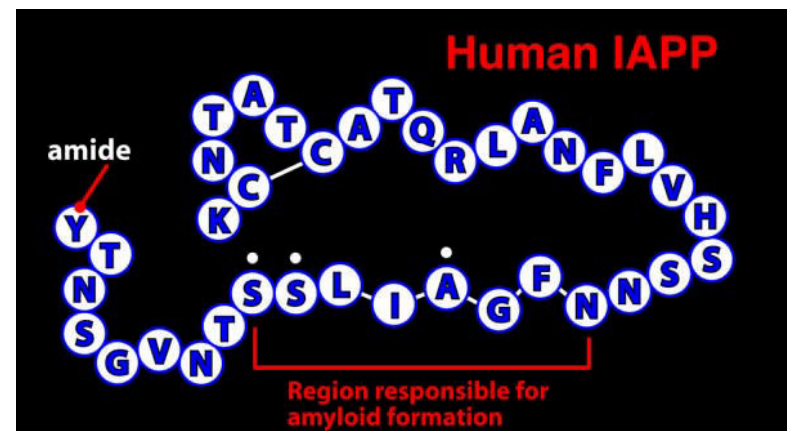

Figure 5:The hIAPP includes amyloidogenic region which is essential for conformational change in the protein structure and disulfide bond at position C2 and C7[20].

Proteins must perfectly fold into three-dimensional structures to be active properly within the cell and organism. Dysfunctional protein aggregation process involves several intracellular events such as misfolding of native protein exposes hydrophobic regions; conformational changes result in unstable intermediates that have a tendency to create oligomers; formation of pathogenic subunits and crossed beta-pleated sheets; and in the T2DM, amyloid fibrils are created with subsequent stabilization by accessory molecules, such as serum amyloid P, perlecan, and apolipoprotein E [57]. When exact folding is unsuccessful, the unfolded, soluble oligomeric proteins start to aggregate, become poisonous, and induce apoptosis [60]. Misfolded IAPP occurs into crossed beta-pleated configurations that are accumulated within the adjacent surrounding extracellular matrix, resulting in space occupying lesions within the islets of the pancreas. Additionally, cellular stressors in T2DM that lead to protein misfolding and aggregation. One of the most essential stressors contributing to this protein conformation aberrancy is redox stress.

When IAPP is unfolded, it tries to become refolded in the ER-Golgi complex, co-occur with the support of ATPdependent chaperone proteins. Kinetically refolding experiments via intermediate proteins related to known conformational diseases have shown that there is an increased energy requirement to accomplish successful refolding due to the high exposure of hydrophobic regions in unfolded or partially-folded proteins. Therefore, exposed hydrogen ions may create a folding pathway to make stably a relative intermediate form of protein that is 'kinetically trapped' if the ER cannot pass this higher energy barrier [61]. When this quality control system is destroyed and IAPP is not able to become correctly refolded, this protein can develop into a soluble toxic monomer due to the innate amyloidogenic properties of the NFGAILSS region of IAPP in amino acid positions 22-29 [62]. Soluble IAPP oligomers have been exhibited to be cytotoxic and possibly responsible for beta cell apoptosis in T2DM. In addition, beta cells with a high replication rate have been observed to be more susceptible to apoptosis by IAPP oligomers [63]. There is the precision balance between refolding and degradation, kept by the quality control system that shows the amount of mutant protein allowed to accumulate. The adaptive and apoptotic mechanisms of the quality control system discriminatory for minor agitation in protein folding efficiency can cause the refusing of nascent IAPP proteins and, subsequently, their accumulation or degradation. Aggregation of mature islet amyloid is responsible for the space filling wound with accompanied secretory and absorptive faults within the islet and is caused to go more rapidly by free radical polymerization due to reactive oxygen species (ROS).

Islet amyloid polypeptide (IAPP) misfolding causes accumulation of proteins. The endoplasmic reticulum (ER), Golgi apparatus, and the lysosome proteasome complex is involved in unfolding and misfolding process of IAPP. The process consists of several arbitrary steps. Firstly, transcription of IAPP from chromosome 12 and translation of IAPP gene occurs. Due to the absence of significant ER stress, chaperones can create proper IAPP. In post-translational modifications IAPP forms a disulfide bond at positions $\mathrm{C} 2$ and $\mathrm{C} 7$, as well as amide formation at the C-terminal tyrosine. The defenselessness of the disulfide bond may be critical feature in the unfolding of IAPP. In the presence of significant ER stress, IAPP will be unfolded and misfolding process will occur. Oligomerization of IAPP is formed. IAPP is transported to the Golgi apparatus. In the absence of significant ER stress normal processing includes co-secretion of normal insulin and IAPP in insulin secretory granules. After unfolding or misfolding, abnormal IAPP is initially delivered into the Golgi apparatus, and in there the misfolded protein undergoes refolding process. If this is not achieved, the misfolded protein then is degraded by the lysosome proteasome complex for into its constituent amino acids. Ubiquitination pathways are also needed to promote trafficking for the lysosome-proteasome complex. When destruction of these organelles occurs, as well as in early T2DM before beta cell failure, the process will end up with apoptosis of the beta cell and the accumulation and aggregation of protofibrils into beta-pleated sheets. In the end, islet amyloid is formed [20].

In the presence of unfavorable conditions, and according to its innative conformational instability, the typically soluble IAPP protein changes its structure to create the crossed beta pleated sheet for aggregation. The propensity of IAPP for aggregation provides an ability to IAPP to endure free radical polymerization which is a process promoted through the cell's reduced capability to clear misfolded proteins. This is the early stage of the pre-diabetic condition [64]. Formed islet amyloid is shown to be quite resistant against the normal proteolytic defenses within the body [65]. Therefore it is permitted to aggregate and achieve rapid free radical polymerization in an islet ambience of increased reactive oxygen species (ROS) [66]. 


\section{Pathogenesis of Protein Misfolding in Type 2 Diabetes}

\section{- $\quad$ Reactive Oxygen Species (ROS)}

Multiple biochemical pathways are involved in the destructive effects of chronic hyperglycemia and oxidative stress on the function of the kidney, retina, vascular tissues and pancreatic islets in Type 2 diabetes [67]. The deleterious potential of oxidative stress on the islets and arterial vessel walls in patients with T2DM is widely accepted. The harmful effects of ROS and other free radicals on proteins, nucleic acids and fats provide a better perception for the amyloid formation within the islets of patients with T2DM. Extravagant redox stress may promote to protein accumulation and aggregation in the ER, with severe consequences for the cell [68].

An ability of ROS to impact disulfide bond formation shows it also influence the development of IAPP misfolding [69]. Newly synthesized proteins in the ER of cells form disulfide bonds which is crucial for proper folding, structure, biological activity, and stability of many secreted and membrane proteins [68,70,71]. Protein folding occurred in the ER of eukaryotesis facilitated by many redox-sensitive chaperones and oxidoreductases such as protein disulfide isomerase, ERp44, ERp72, ERp57, GRP58, Hsp33 [71]. The effects of excessive ROS on native IAPP within the ER may cause covalent breakage of the disulfide bond at positions Cys2 and Cys7 in this 37 amino acid polypeptide, allowing it to unfold or preventing it from properly folding[20].

ROS may affect both proteins and lipids within the ER by changing the ER bilipid layer [72]. Hyperglycemia can cause alterations in the protein content of the ER membrane along formation of advanced glycation end products (AGEs) [73]. When oxidative stress cooperated with hyperglycemia, it changes a protein's susceptibility to glycation[117].In this case, glycation process is to form irreversible Amadori products from reversible Schiff bases with non-enzymatic reactions [74]. These AGEs which tend to aggregate are capable to alter IAPP through posttranslational interactions [75]. With binding of proteoglycan, an augmentation and acceleration are observed in complete amyloid fibril formation. The existence of Amadori products in balance with glucose makes them a clinical marker of glucose control [76].

The processes mentioned above; oxidation and glycation may promote to a dysfunctional ER membrane, meanwhile they allow the aberrant efflux of misfolded proteins into the cytosol before they are accurately formed into their native 3- dimensional structure. This same ER membrane crack may also permit the entrance of ROS into the ER lumen, disrupting the redox sensitive ambient within, and facilitate an even greater unfolding and misfolding of proteins [20].

\section{MATERIALS AND METHODS}

Structural bioinformatics analysis of diabetes associated proteins determined in the Human Diabetes Proteome Project (HDPP)by gathering various datasets over time from healthy and diabetes-diagnosed individuals.

This project was carried out to propose potential protein misfolding with considering IAPP protein as a structural model.

Due to the complex condition of diabetes only a highly interdisciplinary international cooperation can create the multidimensional insights needed to unwind the dynamics of cellular pathways that are key point in diabetes and its related complications. It was purposed to exploit from a worldwide asterism of expertise into a Human Diabetes Proteome Project (HDPP) which is first move to generate systems-level insights into diabetes-associated cellular changes by collecting multivariate datasets over time from specialized cells and organs of healthy and diabetesaffected individuals. The results obtained by the council are available to the wide research community by means of public storage place and data integration platforms such asneXtProt[77].

The HDPP is not only provide extensive information about disease mechanisms but also to determine proteins and isoforms associated with diabetic pathogenesis and complications that are essential for the improvement of better diagnostics, therapies and prevention methods. The generation of HDPP into the inclusive Human Proteome Project(HPP) initiative occurs advantageous conditions for information exchange and combination across all the Chromosome and Biology/Disease HPP initiatives[78].

\section{Data Analysis and Multiple Sequence Alignment} (MSA)

As mentioned above, islet amyloid polypeptide (IAPP), is one of the key proteins in Type 2 diabetes. Since IAPP is secreted protein in which broken sulfide bridge causes to aggregation of this protein in endoplasmic reticulum of beta cells. The function and structure of IAPP protein was studied with using universal protein resource (UniProt) [79].

First IAPP protein sequence was downloaded from UniProt then the region where Disulfide Bridge occur in that sequences was labeled (as it is shown in Figure 1).

1000 diabetes associated protein sequence determined by HDPP was downloaded from UniProt.

Out of 1000, 400 protein's 3-D structure is not analyzed by sophisticated device such as X-ray or NMR. We used computational techniques to obtain their secondary and tertiary structure. Proteins which their structures are analyzed were not in our scope, since those proteins were studied. 400 proteins secondary structure were predicted by one of the most famous predictor PsiPred [80] to get insight about their structural characteristics. 
PsiPred is a tool used for prediction of secondary structure of proteins from the primary sequence by using the information of the evolutionarily related proteins. The prediction process is carried out by three stages which are generation of a sequence profile, prediction of initial secondary structure, and finally the filtering of the predicted structure.

Multiple sequence alignment (MSA) performed among diabetes associated proteins with IAPP protein by using MUSCLE (Multiple Sequence Comparison by LogExpectation) Algorithm [81]. Detected conserved region resulted by MSA was investigated and the residues where disulfide bridge occurs were labeled.

Proteins which have Disulfide Bridge in the conserved sequences were selected for similarity analysis. Both local and global alignments have performed in order to figure out regional and globular similarities in those proteins. Global alignment has been performed by NeedlemanWunsch algorithm which is available in The European Bioinformatics Institute Global Alignment Online Tool[81]. Smith-Waterman algorithm was used for local alignment with using same source.

As a last step, predicted secondary structures of target proteins were analyzed and their 3-D structures were modeled. As mentioned before predicted secondary structures obtained by PSIPRED [80] and 3-D models were analyzed by Swiss-PDB Viewer [82].

Predicted secondary structures were compared with the secondary structure of IAPP and tertiary structures of two similar proteins were illustrated in PDB viewer.

400 proteins were searched to check presence of Disulfide Bridge by using UniProt. Out of 400, 14 proteins were found to have Disulfide Bridge in regions which are similar to disulfide bridge region in IAPP through by MSA and UniProt. Selected 14 proteins are shown in the table1 at below:

\begin{tabular}{|c|c|c|c|}
\hline & Plasminogen-like & & \\
\hline & protein B & $49-73,53-61$ & Secreted \\
\hline & & $177-190,184-199$, & \\
& & $201-212,218-228$, & \\
& & $224-237,239-252$, & \\
& EGF-containing & $258-268,264-277$, & Secreted \\
& fibulin-like & $379-292,298-309$, & \\
& extracellular matrix & $305-318,320-332$, & \\
& protein 1 & $338-350,344-359$, & \\
& & $365-377$ & \\
\hline & & & Secreted \\
\hline
\end{tabular}

Table 1: Selected 14 proteins include regions at which disulfide bridge occur.

\begin{tabular}{|c|c|c|c|}
\hline $\begin{array}{l}\text { UniProt } \\
\text { Codes }\end{array}$ & Protein Name & $\begin{array}{l}\text { Residue Number at } \\
\text { which Disulfide } \\
\text { Bridge Occurs } \\
\end{array}$ & Secreted \\
\hline P06881 & $\begin{array}{l}\text { Calcitonin Gene } \\
\text { Related Peptide } 1\end{array}$ & $84-89$ & Secreted \\
\hline P01185 & $\begin{array}{l}\text { Vasopressin- } \\
\text { neurophysin 2- } \\
\text { copeptin }\end{array}$ & $\begin{array}{c}20-25,41-85,44-58,52- \\
75,59-65,92-104,98- \\
116,105-110\end{array}$ & Secreted \\
\hline P08861 & $\begin{array}{c}\text { Chymotrypsin-like } \\
\text { elastase family } \\
\text { member }\end{array}$ & $\begin{array}{c}58-74,117-120,157- \\
223,188-204,213-244\end{array}$ & - \\
\hline O60500 & Nephrin & $\begin{array}{c}53-111,160-217,265- \\
317,361-417,465- \\
528,567-623,761- \\
816,863-920\end{array}$ & - \\
\hline P10909 & Clusterin & $\begin{array}{c}102-313,113-305 \\
116-302,121-295 \\
129-185\end{array}$ & Secreted \\
\hline O60565 & Gremlin-1 & $\begin{array}{c}94-144,108-158,118- \\
176,122-178\end{array}$ & Secreted \\
\hline P30988 & Calcitonin receptor & $\begin{array}{c}73-99,90-130,113- \\
152\end{array}$ & - \\
\hline P32241 & $\begin{array}{l}\text { Vasoactive } \\
\text { intestinal } \\
\text { polypeptide } \\
\text { receptor } 1\end{array}$ & $\begin{array}{c}50-72,63-105,86-122, \\
215-285\end{array}$ & - \\
\hline P51575 & $\begin{array}{c}\mathrm{P} 2 \mathrm{X} \text { purinoceptor } \\
1 \\
\end{array}$ & $\begin{array}{c}117-165,126-149, \\
132-159,217-227, \\
261-270\end{array}$ & - \\
\hline P56704 & Protein Wnt-3a & $\begin{array}{c}77-88,128-136,138- \\
155,203-217,205- \\
212,297-312,327- \\
342,329-339,334-335 \\
\end{array}$ & Secreted \\
\hline P80370 & $\begin{array}{l}\text { Protein delta } \\
\text { homolog } 1\end{array}$ & $\begin{array}{c}26-37,30-43,45-54 \\
57-68,63-74,76-85 \\
92-103,97-113,115- \\
124,131-144,138- \\
156,158-167,174- \\
185,179-194,196- \\
205,212-223,217- \\
233,235-244\end{array}$ & - \\
\hline
\end{tabular}

Out of 400 proteins, 14 proteins are selected as targets because of their disulfide bridges occur in the conserved region. Both global and local pairwise alignment was performed between each of 14 proteins with IAPP. One of the proteins, Calcitonin Gene Related Peptide 1, was detected as a most similar protein with IAPP. It can be shown as a candidate protein which create conformational change in its structure and cause type II diabetes by taking IAPP as a model. Results are shown in Figure 7 and 8.Calcitonin Gene Related Peptide 1 forms Disulfide Bridge between Cys 84 and Cys89 residues just like Cys2 and Cys7 residue of IAPP. MSA shows that disulfide bridges occur in conserved region. 


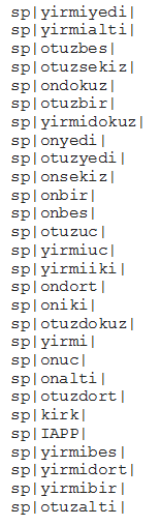

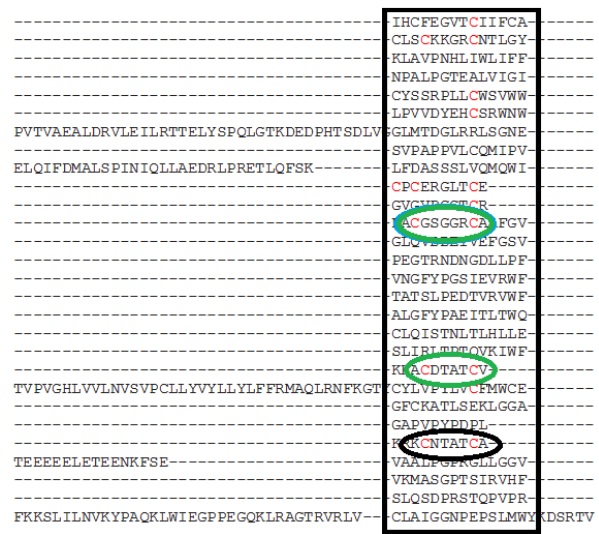

Figure 6: Green circle indicates protein which has disulfide bridge in the conserved sequences in MSA where black circle indicates occurrence of the disulfide bond in IAPP.
Figure 7: Global alignment between IAPP and Calcitonin Gene Related Peptide 1

$$
\begin{aligned}
& \text { IAPP_HUMAN } 10 \text { LIVLSVALNH--LRATPIESHQ--------VERRRCNTATCATQRLANFL } 49
\end{aligned}
$$

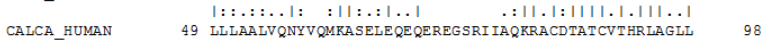

$$
\begin{aligned}
& \text { IAPP_HUMAN } 50 \text { VHS----SNNFGAILSSTNVGSNTYGRR } 73
\end{aligned}
$$

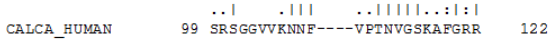

Figure 8: Local alignment between IAPP and Calcitonin Gene Related Peptide 1

After all, secondary and tertiary structures of these two proteins IAPP and Calcitonin Gene Related Peptide 1 were compared for similarity analysis. As it is shown in Figure 9,10 and 11, both secondary and tertiary structures of two proteins consist of fully alpha helix component and contain disulfide bridges. Higher similarity of detected protein is supported by obtained structural analysis results by PsiPred.

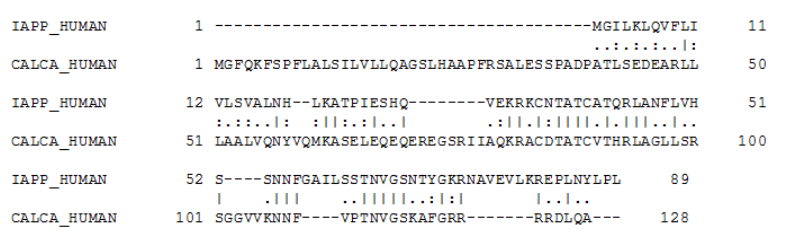

Secondary Structure Map

Feature predictions are colour coded onto the sequence according to the sequence feature key shown below.

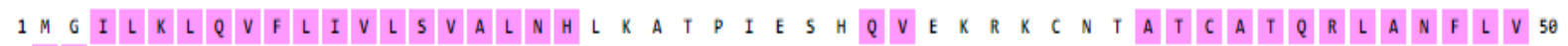

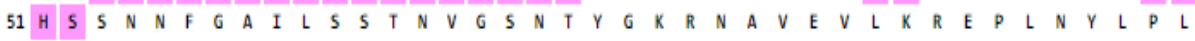

\begin{tabular}{|c|c|c|c|c|c|c|}
\hline KEY & Helix & Sheet & Disordered & $\begin{array}{l}\text { Disordered } \\
\text { protein binding }\end{array}$ & $\begin{array}{l}\text { Dompred } \\
\text { Boundary }\end{array}$ & $\begin{array}{l}\text { DomSSEA } \\
\text { Boundary }\end{array}$ \\
\hline Annotations & n & t & 目 & 回 & A & $D$ \\
\hline
\end{tabular}

Figure 9: Secondary structure of IAPP obtained from PsiPred

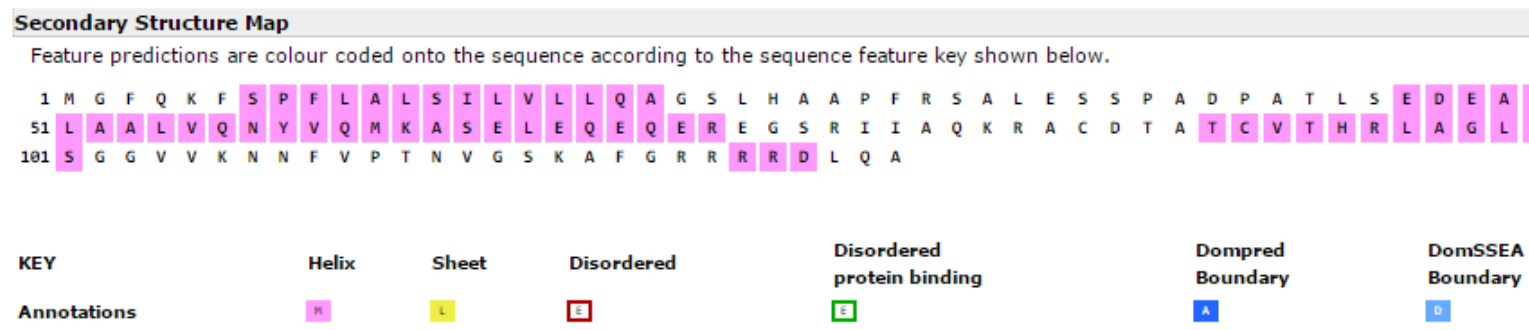

Figure 10: Secondary structure of Calcitonin Gene Related Peptide 1 from PsiPred

Calcitonin Gene Related Peptide 1 (CGRP) shows very similar structural feature with IAPP and it locates outside the cell membrane and secreted hormone like IAPP. The production of CGRP occurs in both peripheral and central neurons. Its abundance of CGRP in the CNS indicates its neurotransmitter or neuromodulator role. CGRP stimulates vasodilatation by enlarging a range of vessels including the coronary, cerebral and systemic vasculature. It also enhances platelet cAMP[148]. As it is described in previous chapter IAPP cannot be secreted due to 
conformational changes in the region where Disulfide Bridge break. Global similarity between these two proteins, occurrence of the disulfide bridge in the conserved region, secondary structure compositions and protein folding patterns, 3D structure of proteins (figure 11 ), show high similarity between these two proteins.

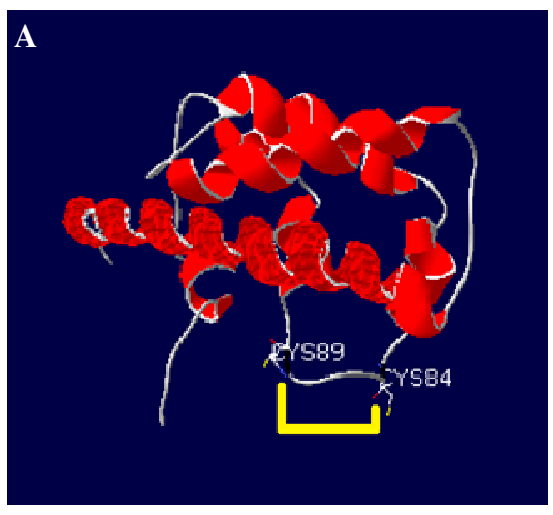

Figure 11: Representation of Calcitonin Gene Related Peptide 1(A) Swiss-PDB viewer [82]. Disulfides bridges are in each models are showed by yellow arrow.

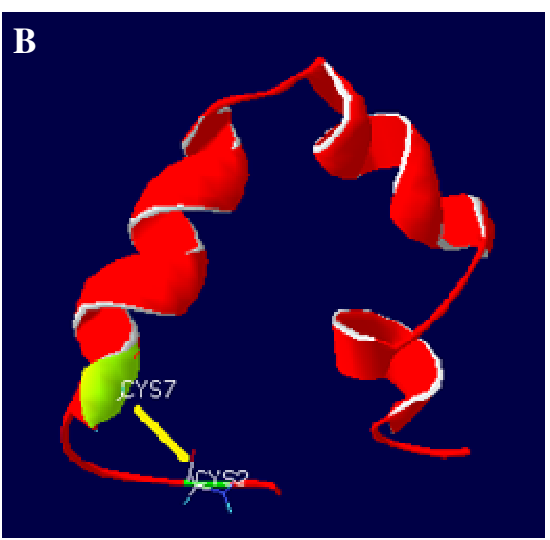

Figure 12: Representation of IAPP by Swiss-PDB viewer [82]. Disulfides bridges are in each models are showed by yellow arrow.

\section{CONCLUSION}

We believe that one of the possible enrolments of the protein Calcitonin Gene Related Peptide 1 to the diabetes can be similar conformational changes due to disulfide bridge formation break.

Finally, our results support that the possible accumulation of harmful, misfolded Calcitonin Gene Related Peptide 1 in pancreatic $\beta$-cells is similar mechanism to other protein based conformational diseases.

Further structural analyses by using sophisticated devices such as X-ray and NMR are needed to be performed due to confirm that conformational changes in Calcitonin Gene Related Peptide 1.

\section{REFERENCES}

1. TA, S. "Diagnosis and classification of diabetes mellitus." Diabetes care 37 (2014): S81.

2. Craig, Maria E., Andrew Hattersley, and Kim C. Donaghue. "Definition, epidemiology and classification of diabetes in children and adolescents."Pediatric diabetes 10.s12 (2009): 312.

3. Galtier, F. "Definition, epidemiology, risk factors." Diabetes \& metabolism 36.6 (2010): 628-651.

4. Piero, M. N. Hypoglycemic effects of some Kenyan plants traditionally used in management of diabetes mellitus in eastern province. Diss. Msc thesis, Kenyatta University, 2006.

5. Reaven, E., et al. "Effect of age and diet on insulin secretion and insulin action in the rat." Diabetes 32.2 (1983): 175-180.

6. Robert, H. "Diabetes Mellitus. Slim Forever International." Diabetes Care (2002), 27-31.

7. Definition, W. H. O. "diagnosis and classification of diabetes mellitus and its complications: report of a WHO consultation." Geneva: World Health Organization (1999).

8. International Expert Committee. "International Expert Committee report on the role of the A1C assay in the diagnosis of diabetes." Diabetes care 32.7 (2009): 1327-1334.

9. American Diabetes Association. "Diagnosis and classification of diabetes mellitus." Diabetes care 32.Supplement 1 (2009): S62-S67.

10. World Health Organization. "Use of glycated haemoglobin $(\mathrm{HbA} 1 \mathrm{c})$ in diagnosis of diabetes mellitus: abbreviated report of a WHO consultation." (2011).

11. Sacks, David B., et al. "Guidelines and recommendations for laboratory analysis in the diagnosis and management of diabetes mellitus." Clinical chemistry 48.3 (2002): 436472.

12. Daneman, Denis. "Type 1 diabetes." The Lancet 367.9513 (2006): 847-858.

13. Hall, John E. Guyton and Hall textbook of medical physiology. Elsevier Health Sciences, 2015 . 
14. Devendra, Devasenan, Edwin Liu, and George S. Eisenbarth. "Type 1 diabetes: recent developments." Bmj 328.7442 (2004): 750-754.

15. 1Couper, Jennifer, and Kim C. Donaghue. "Phases of diabetes in children and adolescents." Pediatric Diabetes 10.s12 (2009): 13-16.

16. Federation, I. D. "International Diabetes Federation: IDF Diabetes Atlas."Brussels, Belgium (2013).

17. Lombardo, F., et al. "Two-year prospective evaluation of the factors affecting honeymoon frequency and duration in children with insulin dependent diabetes mellitus: the key-role of age at diagnosis." Diabetes, nutrition \& metabolism 15.4 (2002): 246-251.

18. Goldberg, Alfred L. "Protein degradation and protection against misfolded or damaged proteins." Nature 426.6968 (2003): 895-899.

19. Oyadomari, Seiichi, Eiichi Araki, and M. Mori. "Endoplasmic reticulum stress-mediated apoptosis in pancreatic $\beta$-cells." Apoptosis 7.4 (2002): 335-345.

20. Camhi, S. L., P. Lee, and A. M. Choi. "The oxidative stress response." New horizons (Baltimore, Md.) 3.2 (1995): 170-182.

21. Westermark, Per. "Quantitative studies of amyloid in the islets of Langerhans." Upsala journal of medical sciences 77.2 (1972): 91-94.

22. Sletten, K. N. U. T., P. Westermark, and J. Boo Natvig. "Characterization of amyloid fibril proteins from medullary carcinoma of the thyroid." The Journal of experimental medicine 143.4 (1976): 993-998.

23. Benditt, E. P., et al. "The major proteins of human and monkey amyloid substance: Common properties including unusual $\mathrm{N}$-terminal amino acid sequences." FEBS letters 19.2 (1971): 169173.

24. Masters, Colin L., et al. "Amyloid plaque core protein in Alzheimer disease and Down syndrome." Proceedings of the National Academy of Sciences82.12 (1985): 4245-4249.

25. Westermark, Per, et al. "A novel peptide in the calcitonin gene related peptide family as an amyloid fibril protein in the endocrine pancreas."Biochemical and biophysical research communications 140.3 (1986): 827-831.

26. Sanke, T., et al. "An islet amyloid peptide is derived from an 89-amino acid precursor by proteolytic processing." Journal of Biological Chemistry 263.33 (1988): 17243-17246.
27. Westermark, Per, et al. "Amyloid fibrils in human insulinoma and islets of Langerhans of the diabetic cat are derived from a neuropeptide-like protein also present in normal islet cells." Proceedings of the National Academy of Sciences 84.11 (1987): 3881-3885.

28. Kayed, Rakez, et al. "Conformational transitions of islet amyloid polypeptide (IAPP) in amyloid formation in vitro." Journal of molecular biology 287.4 (1999): 781-796.

29. Knight, Jefferson D., James A. Hebda, and Andrew D. Miranker. "Conserved and cooperative assembly of membrane-bound $\alpha$ helical states of islet amyloid polypeptide." Biochemistry 45.31 (2006): 94969508.

30. Roh, Jaesook, et al. "Intermedin is a calcitonin/calcitonin gene-related peptide family peptide acting through the calcitonin receptor-like receptor/receptor activity-modifying protein receptor complexes." Journal of Biological Chemistry 279.8 (2004): 7264-7274.

31. 31 Abedini, Andisheh, and Daniel P. Raleigh. "A role for helical intermediates in amyloid formation by natively unfolded polypeptides?." Physical biology 6.1 (2009): 015005 .

32. 32 Westermark, Per, Arne Andersson, and Gunilla T. Westermark. "Islet amyloid polypeptide, islet amyloid, and diabetes mellitus." Physiological reviews 91.3 (2011): 795-826.

33. 33 Watada, Hirotaka, et al. "Involvement of the homeodomain-containing transcription factor PDX-1 in islet amyloid polypeptide gene transcription."Biochemical and biophysical research communications 229.3 (1996): 746-751.

34. Wang, Jing, et al. "The prohormone convertase enzyme 2 (PC2) is essential for processing proislet amyloid polypeptide at the $\mathrm{NH} 2$-terminal cleavage site." Diabetes 50.3 (2001): 534-539.

35. Mulder, H., B. Ahren, and F. Sundler. "Islet amyloid polypeptide and insulin gene expression are regulated in parallel by glucose in vivo in rats."American Journal of PhysiologyEndocrinology And Metabolism 271.6 (1996): E1008-E1014.

36. Rickels, Michael R., Heather W. Collins, and Ali Naji. "Amyloid and transplanted islets." N Engl J Med 359.25 (2008): 2729.

37. Mulder, Hindrik, et al. "Islet amyloid polypeptide is expressed in endocrine cells of the gastric mucosa in the rat and 
mouse." GASTROENTEROLOGY-BALTIMORE THEN PHILADELPHIA- 107 (1994): 712-712.

38. Cooper, G. J., et al. "Amylin found in amyloid deposits in human type 2 diabetes mellitus may be a hormone that regulates glycogen metabolism in skeletal muscle." Proceedings of the National Academy of Sciences 85.20 (1988): 7763-7766.

39. Deems, Rhonda Oetting, Richard W. Deacon, and Douglas A. Young. "Amylin activates glycogen phosphorylase and inactivates glycogen synthase via a cAMP-independent mechanism." Biochemical and biophysical research communications 174.2 (1991): 716-720.

40. Zierath, J. R., et al. "Human islet amyloid polypeptide at pharmacological levels inhibits insulin and phorbol ester-stimulated glucose transport in in vitro incubated human muscle strips." Diabetologia 35.1 (1992): 26-31.

41. Johnson, K. H., et al. "The putative hormone islet amyloid polypeptide (IAPP) induces impaired glucose tolerance in cats." Biochemical and biophysical research communications 167.2 (1990): 507-513.

42. Ludvik, Bernhard, et al. "Increased levels of circulating islet amyloid polypeptide in patients with chronic renal failure have no effect on insulin secretion." Journal of Clinical Investigation 94.5 (1994): 2045.

43. Bretherton-Watt, D., et al. "Very high concentrations of islet amyloid polypeptide are necessary to alter the insulin response to intravenous glucose in man." The Journal of Clinical Endocrinology \& Metabolism 74.5 (1992): 1032-1035.

44. Westermark, Gunilla T. "Endocrine amyloid." Amyloid Proteins: The Beta Sheet Conformation and Disease (2005): 723-754.

45. Buxbaum, Joel N. "Diseases of protein conformation: what do in vitro experiments tell us about in vivo diseases?." Trends in biochemical sciences 28.11 (2003): 585-592.

46. Glenner, G. G., et al. " $\beta$-pleated sheet fibrils a comparison of native amyloid with synthetic protein fibrils." Journal of Histochemistry\& Cytochemistry22.12 (1974): 1141-1158.

47. Hellman, Ulf, et al. "Amino acid sequence from degu islet amyloid-derived insulin shows unique sequence characteristics." Biochemical and biophysical research communications 169.2 (1990): 571-577.

48. Yumlu, Saniye, et al. "Localized insulin-derived amyloidosis in patients with diabetes mellitus: a case report." Human pathology 40.11 (2009): 1655-1660.

49. Nishi, Masahiro, et al. "Conservation of the sequence of islet amyloid polypeptide in five mammals is consistent with its putative role as an islet hormone." Proceedings of the National Academy of Sciences 86.15 (1989): 5738-5742.

50. Westermark, P., et al. "Islet amyloid polypeptide-a novel controversy in diabetes research." Diabetologia 35.4 (1992): 297-303.

51. Westermark, Per, et al. "Islet amyloid polypeptide: pinpointing amino acid residues linked to amyloid fibril formation." Proceedings of the National Academy of Sciences 87.13 (1990): 5036-5040.

52. Betsholtz, Christer, et al. "Sequence divergence in a specific region of islet amyloid polypeptide (IAPP) explains differences in islet amyloid formation between species." FEBS letters 251.1-2 (1989): 261-264.

53. Nilsson, Melanie R., and Daniel P. Raleigh. "Analysis of amylin cleavage products provides new insights into the amyloidogenic region of human amylin." Journal of molecular biology 294.5 (1999): 1375-1385.

54. Dunn, William A. "Autophagy and related mechanisms of lysosome-mediated protein degradation." Trends in cell biology 4.4 (1994): 139-143.

55. Kahn, Steven E., SofianosAndrikopoulos, and C. Bruce Verchere. "Islet amyloid: a longrecognized but underappreciated pathological feature of type 2 diabetes." Diabetes 48.2 (1999): 241-253.

56. Nanga, Ravi Prakash Reddy, et al. "Threedimensional structure and orientation of rat islet amyloid polypeptide protein in a membrane environment by solution NMR spectroscopy." Journal of the American Chemical Society 131.23 (2009): 8252-8261.

57. Butler, Alexandra E., et al. "Increased $\beta$-cell apoptosis prevents adaptive increase in $\beta$-cell mass in mouse model of type 2 diabetes evidence for role of islet amyloid formation rather than direct action of amyloid." Diabetes 52.9 (2003): 2304-2314.

58. Clark, A., et al. "Pancreatic pathology in noninsulin dependent diabetes (NIDDM)." Diabetes research and clinical practice 28 (1995): S39S47.

59. Hayden, M. R. "Hayden MR, Tyagi SC." JOP. J Pancreas (Online) 2 (2001): 124-39. 
60. Chiti, Fabrizio, et al. "Kinetic partitioning of protein folding and aggregation."Nature Structural \& Molecular Biology 9.2 (2002): 137143.

61. Kapurniotu, Aphrodite, AnkeSchmauder, and Konstantinos Tenidis. "Structure-based design and study of non-amyloidogenic, double $\mathrm{N}$ methylated IAPP amyloid core sequences as inhibitors of IAPP amyloid formation and cytotoxicity." Journal of molecular biology 315.3 (2002): 339-350.

62. Janson, Juliette, et al. "The mechanism of islet amyloid polypeptide toxicity is membrane disruption by intermediate-sized toxic amyloid particles."Diabetes 48.3 (1999): 491-498.

63. Gaudet, Pascale, et al. "neXtProt: organizing protein knowledge in the context of human proteome projects." Journal of proteome research 12.1 (2012): 293-298.

64. Badman, M. K., et al. "Fibrillar islet amyloid polypeptide (amylin) is internalised by macrophages but resists proteolytic degradation." Cell and tissue research 291.2 (1998): 285-294.

65. Koning, Eelco JP, et al. "Macrophages and pancreatic islet amyloidosis."Amyloid 5.4 (1998): 247-254.

66. UniProt Consortium. "UniProt: a hub for protein information." Nucleic acids research(2014): gku989.

67. Kopito, Ron R., and David Ron. "Conformational disease." Nature Cell Biology 2.11 (2000): E207E209.

68. Cumming, Robert C., et al. "Protein disulfide bond formation in the cytoplasm during oxidative stress." Journal of Biological Chemistry 279.21 (2004): 21749-21758.

69. Anelli, Tiziana, et al. "ERp44, a novel endoplasmic reticulum folding assistant of the thioredoxin family." The EMBO journal 21.4 (2002): 835-844.

70. Fassio, Anna, and Roberto Sitia. "Formation, isomerization and reduction of disulphide bonds during protein quality control in the endoplasmic reticulum."Histochemistry and cell biology 117.2 (2002): 151-157.

71. Hayden, Melvin R., et al. "Type 2 diabetes mellitus as a conformational disease." Jop 6.4 (2005): 287-302.

72. Yue, D. K., S. McLennan, and J. R. Turtle. "Nonenzymatic glycosylation of tissue protein in diabetes in the rat." Diabetologia 24.5 (1983): 377-381.
73. Schnider, Stuart L., and R. R. Kohn. "Glucosylation of human collagen in aging and diabetes mellitus." Journal of Clinical Investigation 66.5 (1980): 1179.

74. BROWNLEE, MICHAEL, HELEN VLASSARA, and ANTHONY CERAMI. "Nonenzymatic glycosylation and the pathogenesis of diabetic complications." Annals of internal medicine 101.4 (1984): 527-537.

75. Ma, Zhi, Per Westermark, and Gunilla T. Westermark. "Amyloid in human islets of Langerhans: immunologic evidence that islet amyloid polypeptide is modified in amyloidogenesis." Pancreas 21.2 (2000): 212218.

76. Koenig, RONALD J., STEVEN H. Blobstein, and A. N. T. H. O. N. Y. Cerami. "Structure of carbohydrate of hemoglobin AIc." Journal of Biological Chemistry 252.9 (1977): 2992-2997.

77. HUPO. Human Proteome Project (HPP). Available

from:http://www.hupo.org/research/hpp/

78. Paik Y-K. "The Chromosome-Centric Human Proteome Project for cataloging proteins encoded in the genome." Nature Biotechnology 30.3 (2012): 221-3.

79. Robertson, R. Paul. "Chronic oxidative stress as a central mechanism for glucose toxicity in pancreatic islet beta cells in diabetes." Journal of Biological Chemistry 279.41 (2004): 4235142354.

80. Guex, Nicolas, and Manuel C. Peitsch. "SWISS-MODEL and the Swiss-Pdb Viewer: an environment for comparative protein modeling." electrophoresis18.15 (1997): 27142723.

81. Kitamura, Kazuo, et al. "Isolation and characterization of peptides which act on rat platelets, from a pheochromocytoma." Biochemical and biophysical research communications 185.1 (1992): 134-141.

82. http://www.ebi.ac.uk/Tools/msa/ 